\title{
Silicone Gel Bleed on Breast Implants
}

\author{
Felipe Rebello Lourenço", Irene Satiko Kikuchi and Terezinha de Jesus Andreoli Pinto
}

Faculty of Pharmaceutical Sciences, University of São Paulo, Brazil

\begin{abstract}
Silicone is a polymer widely used for medical purposes, including breast implants. The aim of this work is to verify the occurrence of silicone gel diffusion through the lining membrane (bleed) of gel breast implants with smooth, textured, and polyurethane foam coated surfaces, sterilized by dry heat and ethylene oxide. The results enable us to conclude that there is significant difference for the bleed results among gel breast implants of smooth, textured, and polyurethane foam coated surfaces in relation to the bleed test. The bleed results for implants sterilized by dry heat and ethylene oxide also show significant difference. These results suggest that the gel diffusion through the lining membrane is related to the type of gel breast implants, as well as to the sterilization method. It means that polyurethane foam coated surface breast implant shows less gel diffusion than textured and smooth breast implants and breast implants sterilized by dry heat show less gel diffusion than those sterilied by ethylene oxide.
\end{abstract}

Keywords: Breast implants, silicone, bleed.

\section{INTRODUCTION}

Silicone is a polymer used in varied medical applications, including breast implants. Intra-capsular leakage to the surrounding tissue and circulation is potentially toxic due to the development of cell and humoral response. The silicone gel implant migration has been appointed as the main cause of capsular contracture [1-6].

The capsular contracture results from a foreign body type of reaction, with the isolation of the implant from the tissue in which it is contained by a fibrous capsule, what can result in breast hardening, thus altering the shape softness and the consistence smoothness, in same cases leading to a new surgery [7-13].

Sterility is a demand in which concerns gel silicone implants and it can be achieved by physical and chemical methods. Among physical sterilization processes, dry heat is rather effective. However, it can lead to silicone gel hydrolysis and consequent damage of the product characteristics, as it is performed at high temperature. Chemical sterilization employing ethylene oxide offers the advantage of effective treatment at relatively low temperatures. However, gas residue, alkylate agents, can react with silicone functional groups, altering its properties [14-19].

Thus the aim of this work is to verify the occurrence of silicone gel diffusion through the lining membrane of gel breast implants of smooth (smooth), textured (text), and polyurethane foam coated (CPU) surfaces, sterilized by dry heat (heat) and ethylene oxide (ETO).

*Address correspondence to this author at the Faculty of Pharmaceutical Sciences, University of São Paulo, Brazil; Tel: 55-11-3091-2218; Fax: 5511-3091-3626; E-mail: feliperl@usp.br

\section{MATERIAL AND METHODS}

The bleed test was performed according to ASTM F70396 norm [20], employing silicone discs with a diameter of 50 mm (surface area of $19,635 \mathrm{~cm}^{2}$ ), thickness of $3,125 \mathrm{~mm} \pm$ $0,025 \mathrm{~mm}$ and hardness of $70 \mathrm{ShA}$, as a support for the implants. 60 gel breast implants with smooth, textured, and coated with polyurethane foam surfaces (Fig. 1), sterilized by dry heat and ethylene oxide, were assayed (Table 1). ASTM F703-96 norm covers the requirements for silicone gel filled, saline inflatable silicone gel filled, and saline inflatable, smooth shell implantable breast prostheses intended for use in surgical reconstruction, augmentation, or replacement of the breast.

The silicone discs were previously clean with isopropyl alcohol, being afterwards kept in incubator (Nova Ética, Mod. $411 \mathrm{D}, \pm 0,1{ }^{\circ} \mathrm{C}$ ) for 12 hours, at $43,0 \pm 2,0{ }^{\circ} \mathrm{C}$, for steadiness, being then weighed in analytical scales (Mettler Toledo, AG3000, $\pm 0,1 \mathrm{mg}$ ).

After implant cleaning with isopropyl alcohol and room temperature drying for 2 hours, the implants were placed on the silicone discs (Fig. 2) and put in an oven at 43,0 $\pm 2,0^{\circ} \mathrm{C}$ for a week, after which the first weighing procedure took place.

After this period the control and support discs were removed from the incubator and left at room temperature for steadiness for 1 hour. They were then weighed on analytical scales, being afterwards replaced in the incubator with the implants deposited on the support discs. This procedure was repeated for 8 consecutive weeks. The assay was performed in a room with controlled temperature and relative humidity $\left(26,0 \pm 3,0{ }^{\circ} \mathrm{C}\right.$ and $\left.62 \pm 16 \% \mathrm{UR}\right)$.

\section{RESULTS}

The results of the bleed test, performed in an 8-week period, for gel breast implants with smooth, textured, and 
polyurethane foam coated surfaces, sterilized by dry heat and ethylene oxide, are shown in the graph below (Fig. 3).

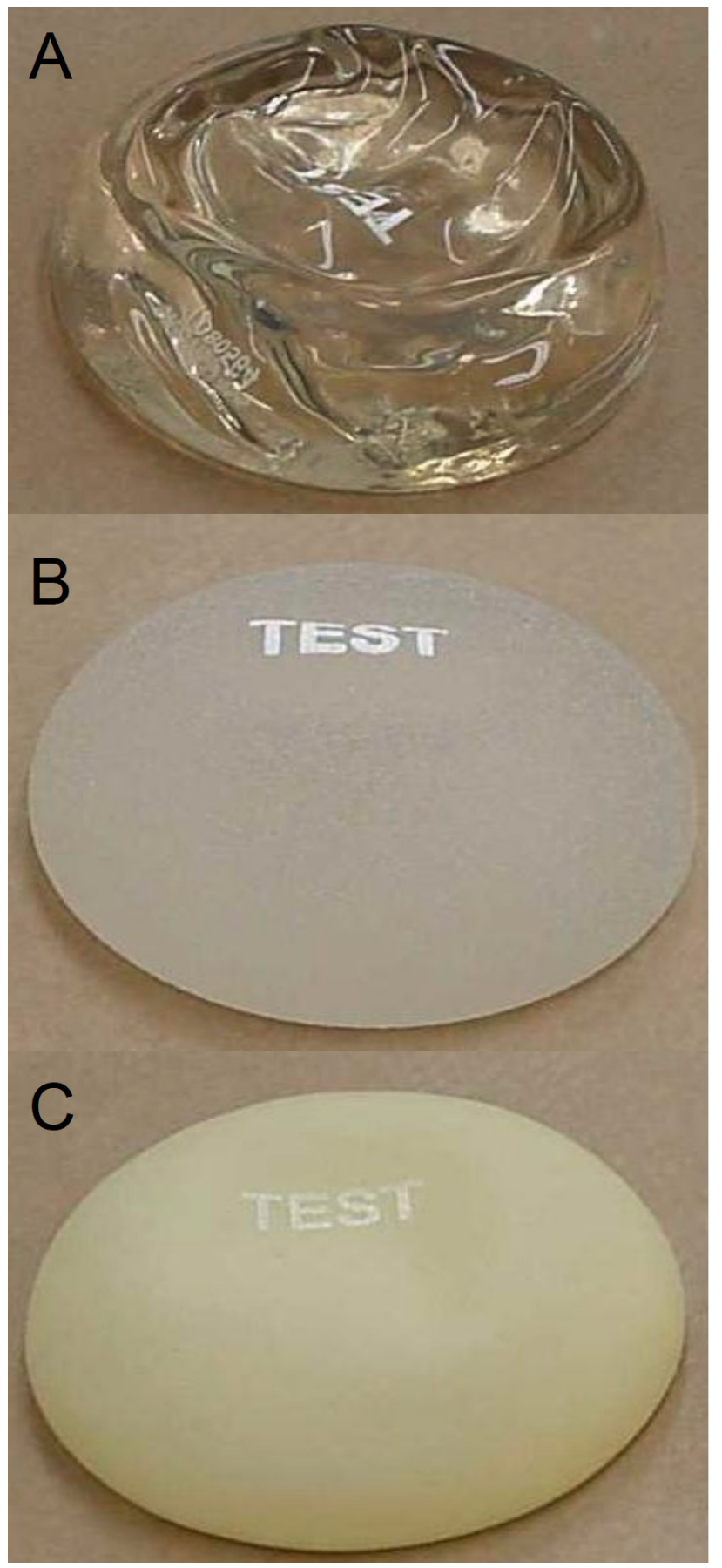

Fig. (1). Gel breast implants with smooth surface (A), textured surface $(\mathbf{B})$ and polyurethane foam coated surface $(\mathbf{C})$.

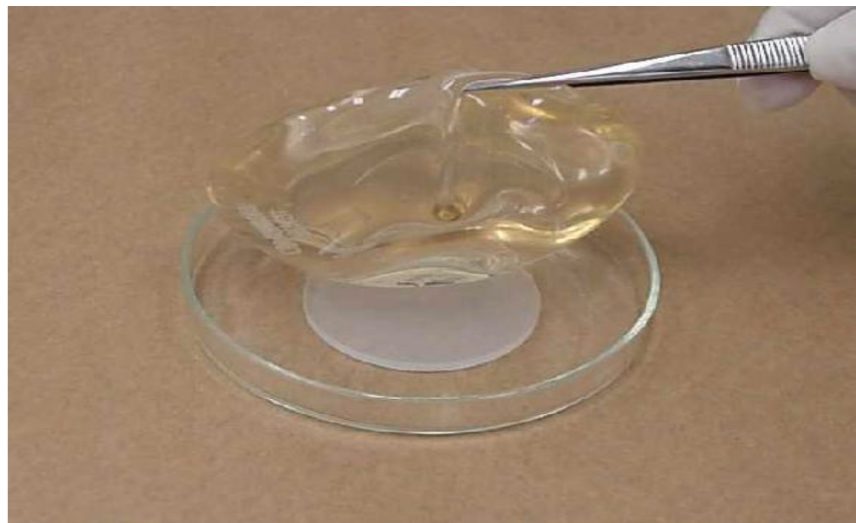

Fig. (2). Gel breast implant and silicone disc.

The variance analysis (Fig. 4) allows us to conclude that there is significant difference $(p=0,000)$ between the results obtained for the breast gel implants of smooth, textured, and polyurethane foam coated surfaces in relation to bleed test. Moreover there is also significant difference $(\mathrm{p}=0,000)$ between the results obtained for gel breast implants sterilized by dry heat and ethylene oxide relatively to the bleed test.

The results indicates that the gel "bleed" is more affect by the type of surface affects than by the sterilization process. Smooth surface breast implants showed a gel "bleed" of $1,0455 \mathrm{mg} / \mathrm{cm}^{2} /$ week against 0,3377 $\mathrm{mg} / \mathrm{cm}^{2} /$ week for textured surface breast implants and $0,1752 \mathrm{mg} / \mathrm{cm}^{2} /$ week for polyurethane foam coated surface.

Breast implants sterilized by dry heat showed gel "bleed" of $0,47695 \mathrm{mg} / \mathrm{cm}^{2} /$ week against $0,56202 \mathrm{mg} / \mathrm{cm}^{2} /$ week for those sterilized by ethylene oxide. Although these values are not so distant, if we evaluate separately each type of surface, the breast implants sterilized by dry heat always showed lower gel "bleed" than those sterilized by ethylene oxide, as we can observe in Fig. (3).

\section{DISCUSSION}

The evaluation of the amount of gel diffused by the implants is fundamental, due to the fact that this is the main cause of capsular contracture. Since the first procedures of breast implants several modifications have taken place with a view to the decrease of capsular contracture incidence. However implant evolution has not been paralleled by the development of methodologies to evaluate these materials. Several studies have been carried out to evaluate the safety of implants, mainly involving explants or adjacent tissues to the implants. Nevertheless there is lack of methods to evaluate the rate of gel silicone migration, as the only procedure described under international norm, despite supplying orientation in what concerns comparative studies

Table 1. Numbers Gel Breast Implants Assayed for Bleed Test

\begin{tabular}{|c|c|c|c|}
\hline Type of Gel Breast Implants & Dry Heat Sterilization & Ethylene Oxide Sterilization & Total \\
\hline smooth surface & 10 & 10 & 20 \\
\hline textured surface & 10 & 10 & 20 \\
\hline coated with polyurethane foam surface & 10 & 10 & 20 \\
\hline Total & 30 & 30 & 60 \\
\hline
\end{tabular}




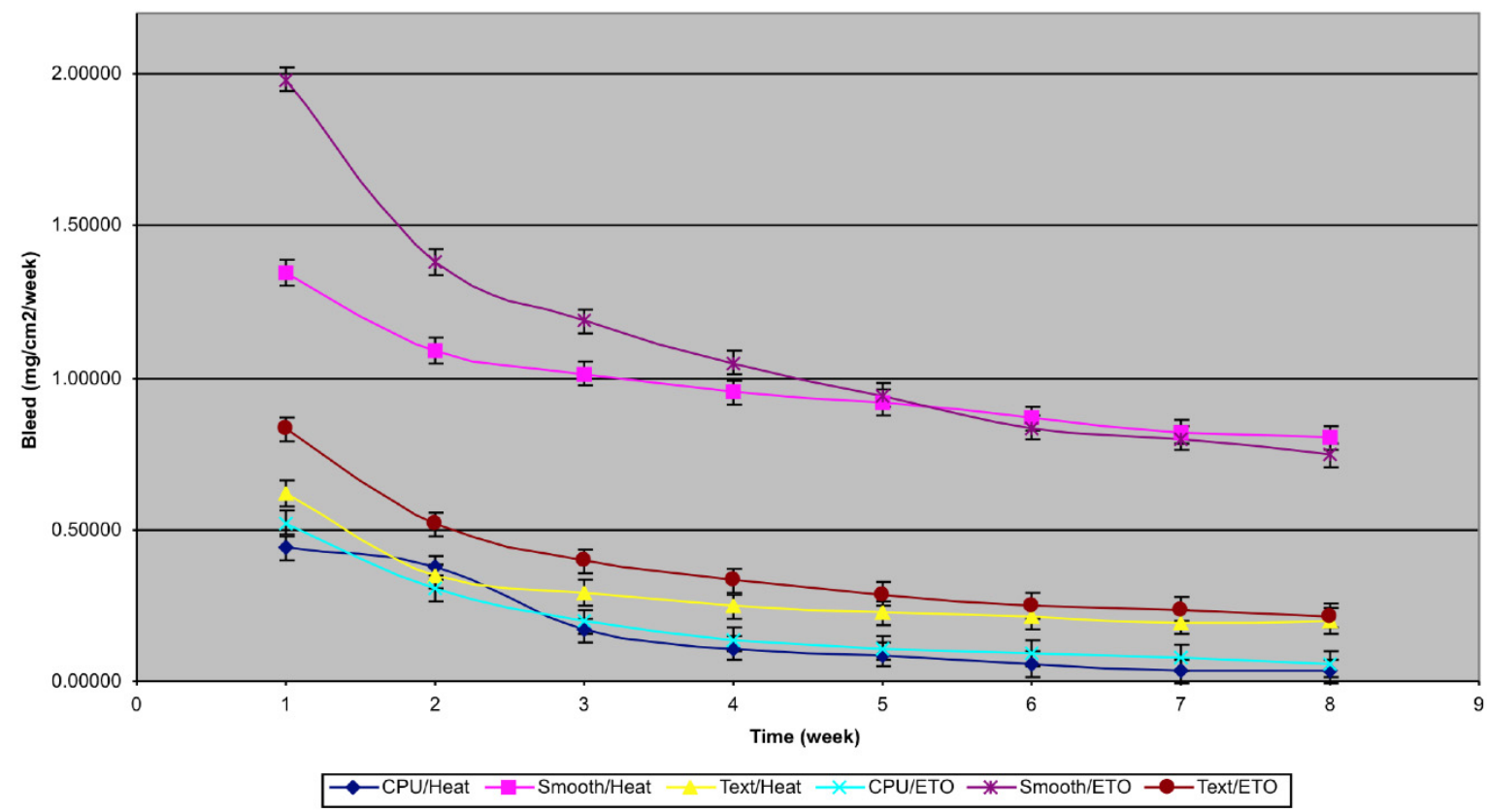

Fig. (3). Results of bleed test for gel breast implants with smooth, textured and polyurethane foam coated surfaces, sterilized by dry heat and ethylene oxide.

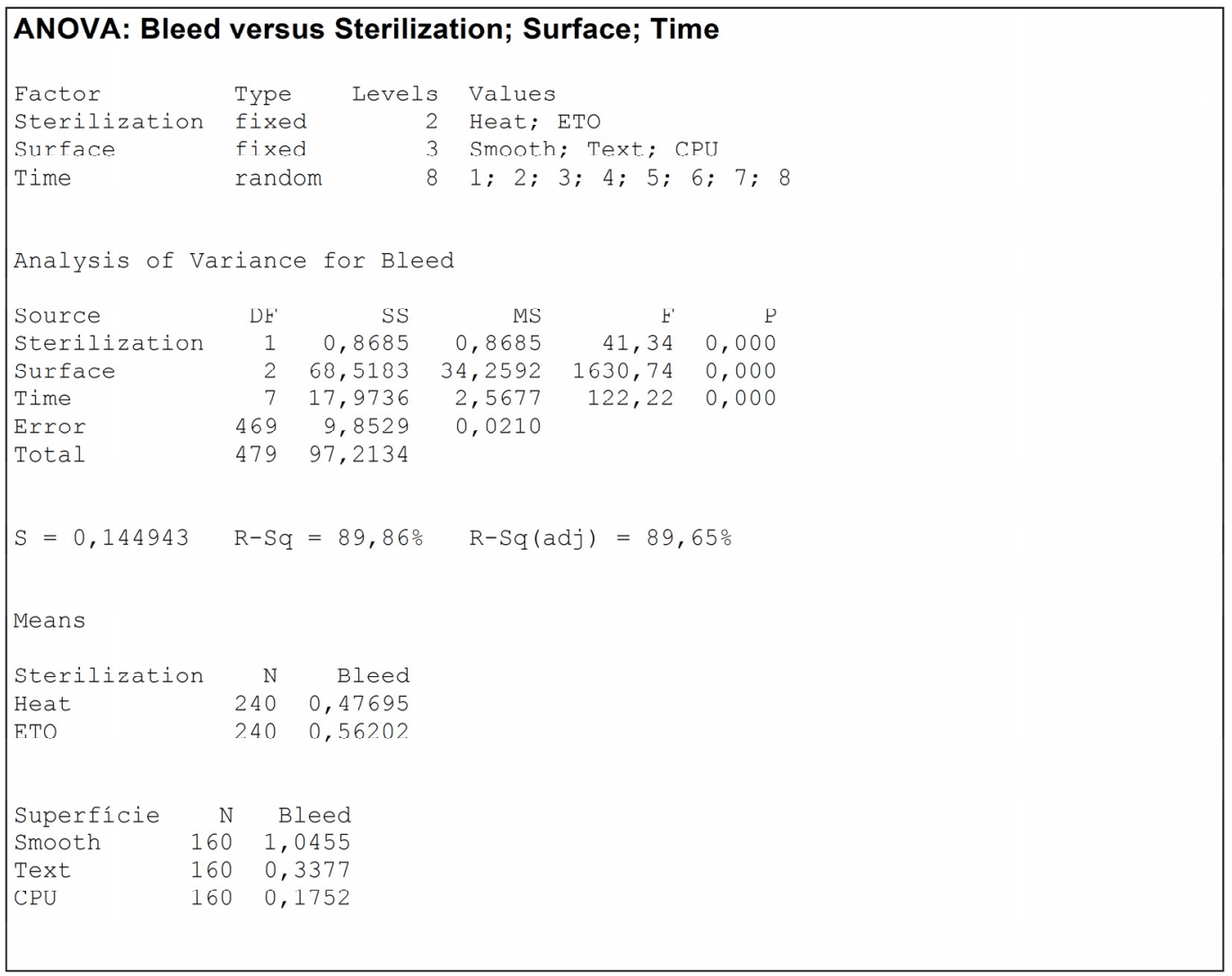

Fig. (4). Analisys of Variance (ANOVA) of bleed test for gel breast implants with smooth, textured and polyurethane foam coated surfaces, sterilized by dry heat and ethylene oxide.

between different types of implants, does not give specifications as to the acceptable rate of silicone gel migration.

The Guidance for Industry and FDA Staff - Saline, Silicone Gel, and Alternative Breast Implants, from 17
November 2006, recommends the study of silicone gel migration employing silicone implants immersed in media which simulate the composition of human interstitial fluids [21]. The medium must be changed, simulating an in vivo dynamic condition and the test must be carried out until gel 
constituents can be observed in the medium. The Guide also states that the constituents should be identified, as well as the respective rates of migration and the variance of the latter throughout time. The proposed modification is effectively a better simulation of physiological conditions than the method adopted in this work. However its application is not possible due to the lack of information that can, moreover, demonstrate the advantages of its use.

The ASTM F703-96 [20] method employed in this work does not supply information about the nature of the material that migrates from the silicone implants. However the results obtained give important information concerning the amount of gel diffused in relation to the sterilization method and type of coating of the implants. Smooth implants present higher level gel diffusion than textured surface ones, showing the latter a higher level of diffusion than the polyurethane foam coated ones. Also, implants sterilized by dry heat show minor gel diffusion compared with those sterilized by ethylene oxide.

The inflammatory potential risk of silicone breast implants has focused on the gel "bleed" through the membrane [4]. Since the silicone gel and the membrane are manufactured from the same materials, the membrane is not impermeable to its contents [4]. So, breast implants with lower gel "bleed" may show less inflammatory potential.

Smooth breast implants were the firsts silicone gel-filled implants used in aesthetic surgery [22]. This type of breast implant show a higher incidence of post-surgery problems. In the 70's the firsts breast implants covered with a thin layer of polyurethane foam were developed. Polyurethane-covered implants show a lessened incidence of capsular contracture [22]. One of the reasons for this lower toxicity of covered implants may be explained due to a lower gel "bleed" of this implants. Introduction of barriers layers may improve the smooth surface membrane to lower the gel "bleed" in this kind of breast implant.

Animal studies showed that higher molecular weight silicone are more reactive than lower molecular weight silicone [4]. The method employed in this study do not allow the evaluation of molecular weight of the silicone that "bleed" through the membrane. However, we can concluded that there are significant difference of gel "bleed" among the types of breast implants evaluated.

These results suggest that the diffusion of gel through the coating membrane (bleed) is related to the type of surface gel breast implants have, as well as to the sterilization method employed.

\section{REFERENCES}

[1] Brand KG. Polyurethane-coated silicone implants and the question of capsular contracture. Plast Reconst Surg 1984; 73: 498.

[2] Busch H. Silicone toxicology. Sem Arthr Rheum 1994, 24(1 Suppl): 11-7.

[3] O'Neill JK, Taylor GI. A novel method to remove silicone gel after breast implant rupture. J Plastic Reconst Aesthet Surg 2006; 59(8): 889-91.

[4] Yoshida SH, Swan S, Teuber SS, Gershwin E. Silicone breast implants: immunotoxic and epidemiologic issues. Life Sci 1995; 56(16): 1299-371.

[5] Basile AR, Basile F, Basile AVD. Late infection following breast augmentation with textured silicone gel-filled implants. Aesthet Surg J 2005; 25(3): 249: 54 .

[6] Collins N, Coleman D, Foo I. Ten-year review of a prospective randomized controlled trial of textured versus smooth subglandular silicone gel breast implants. Plas Reconst Surg 2000; 106: 786-91.

[7] Gerstzen P. A formal risk assessment of silicone breast implants. Biomaterials 1999; 20: 1063-69.

[8] Lahiri A, Waters R. Locoregional silicone spread after high cohesive gel silicone implant rupture. J Plastic Reconst Aesthet Surg 2006; 59(8): 885-6.

[9] Dreyer JM. Cohesive gel implants for breast augmentation. Aesthet Surg J 2003; 23(5): 405-9.

[10] Fergusson JH. Silicone breast implants and neurologic disorders: report of a practice committee of the American academy of neurology. Neurology 1997; 58: 1504-7.

[11] O'Toole M, Caskey CI. Imaging spectrum of breast implant complications: mammography, ultrasound, and magnetic resonance imaging. Sem Ultrasound CT MRI 2000; 21(5): 351-61.

[12] Smahel J. Tissue reactions to breast implant coated with polyurethane. Plast Reconst Surg 1978; 61: 80-5.

[13] Topez M, Habal MB. The use of fatty and hydrogels as filling material in breast implants. Oper Techn Plastic Reconst Surg 2002; 9(2): 79-81.

[14] Athanasiou KA, Niederauer GG, Agrawal CM. Sterilization, toxicity, biocompatibility and clinical applications of polyactic acid/polyglicolic acyd copolymers. Biomaterials 1996; 17: 93-102.

[15] Gogolewski S, Mainil-Varlet P. The effect of thermal treatment on sterility molecular and mechanical properties of various polyactides. Biomaterials 1996; 17: 523-8.

[16] International Organization for Standardization. ISO 14607: Implants for surgery-specific requirements for mammary implants. Geneva: ISO 2002; p. 16.

[17] Marreco PR, Moreira PL, Genari SC, Moraes AM. Effects of different sterilization methods on the morphology, mechanical, properties, and cytotoxicity of chitosan membranes used as wound dressings. J Biomed Mater Res B Appl Biomater 2004; 71: 268-77.

[18] Pinto T de JA, Cruz AS, Azevedo JC de. Implantes mamários de silicone: efeitos dos métodos de esterilização sobre a migração de gel de silicone e biocompatibilidade. Bio Farma-Rev Tec Cient Farm Bioquim Anal Clin Toxicol 2006; 1(1): 9-14.

[19] Takechi M, Miyamoto Y, Momota Y, et al. Effects of various sterilization methods on the setting and mechanical properties of apatite cement. J Biomed Mater Res B Appl Biomater 2004; 69: 99-103.

[20] American Society for Testing Materials. Standard specifications for implantable breast prosthesis: F703-96. Philadelphia: ASTM, 2002, 9 p.

[21] Guidance for Industry and FDA Staff - Saline, Silicone Gel, and Alternative Breast Implants. FDA, 2006; p. 40.

[22] Maxwell GP, Gabriel A. The evolution of breast implants. Clin Plasctic Surg 2009; 36: 1-13. 